\title{
Redes de apoyo a familias en Tunja durante la pandemia: una respuesta ante la crisis generada por la COVID-19
}

\author{
Support networks for families in Tunja during the pandemic: a \\ response to the crisis generated by COVID-19
}

Haider Esteban Bautista Joaqui ${ }^{1 *}$ (르 ; Junior Adrián Franco Riaño ${ }^{(\mathbb{D} ;}$; Leidy Johana Mendivelso Vargas ${ }^{3}$ (i)

Recibido: 23-09-2020; Aceptado: 12-12-2020; Publicado: 28-12-2020

\section{RESUMEN}

Objetivo. La presente investigación tiene como objetivo conocer las redes de apoyo presentes en familias de Tunja durante la pandemia generada por la COVID-19, así como indagar qué es para las familias una red de apoyo e identificar cuáles son las redes de apoyo y el tipo de apoyo que ellos consideran que deberían estar más presentes en tiempo de pandemia. Método. Se desarrolla bajo un enfoque mixto, para obtener mayor detalle en la información recolectada mediante el uso de una encuesta que fue aplicada en un formulario creado en Google Forms y compartido mediante redes sociales a través de un muestreo por conveniencia a 119 familias de la ciudad de Tunja, con diversas características sociodemográficas, donde sus jefes de hogar oscilaban entre los 19 y 65 años y se encontraban entre estratos socioeconómicos del 1 al 5. Resultados. Los principales hallazgos evidencian que, durante la pandemia, las principales redes de apoyo a familias tunjanas son otros familiares y amigos. El apoyo que más recibieron es el emocional, resultados que concuerdan con las demandas de los mismos participantes en situaciones similares. Las familias participantes le asignan el significado al termino red de apoyo, muy ligado a las características de una red informal. Más de la mitad de la muestra desconoce redes de apoyo a familias, y mencionan con mayor frecuencia a instituciones religiosas y organizaciones gubernamentales.

Palabras clave: Red de apoyo; tipo de apoyo; familia; pandemia; COVID-19; familia extensa; amigos; instituciones; religión; vecinos; apoyo emocional; apoyo informativo; apoyo tangible; significado; fortalezas, dificultades.

\section{ABSTRACT}

Objective. The present research aims to know the support networks present in Tunja families during the pandemic generated by COVID-19, as well as to investigate what a support network is for families and identify which are the support networks and the type of support that they consider should be more present in times of pandemic. Method. It is developed under a mixed approach, to obtain greater detail in the information collected by a survey that was applied in a form created

\footnotetext{
${ }^{1}$ Fundación Universitaria Juan de Castellanos, Trabajo Social. Grupo de Investigación Ciclo Vital, Familia y Desarrollo Humano. Tunja. hbautista@jdc.edu.co ${ }^{2}$ Fundación Universitaria Juan de Castellanos, Trabajo Social. Grupo de Investigación Ciclo Vital, Familia y Desarrollo Humano. Tunja. jafranco@jdc.edu.co ${ }^{3}$ Fundación Universitaria Juan de Castellanos. Tunja. 1mendivelso@jdc.edu.co respuesta ante la crisis generada por la COVID-19. Búsqueda, v. 7, n. 25, e496. https://doi.org/10.21892/01239813.496
} 
in Google Forms and shared through social networks through a convenience sampling to 119 families of the city of Tunja, with various sociodemographic characteristics, where their heads of household ranged between 19 and 65 years of age and were between socioeconomic strata from 1 to 5. Results. The main findings show that, during the pandemic, the main networks of support to tunjanas families are other relatives and friends. The support they received the most was emotional, results that were consistent with the demands of the same participants in similar situations. The participating families assign the meaning to the term support network, closely linked to the characteristics of an informal network. More than half of the sample is unaware of family support networks, and they mention religious institutions and government organizations more frequently.

Keywords: Support network; type of support; family; pandemic; COVID-19; extended family; friends; institutions; religion; neighbors; Emotional Support; informational support; tangible support; meaning; strengths, difficulties.

\section{INTRODUCCIÓN}

A finales del año 2019, en Wuhan, ciudad de China, se reportó el aumento alarmante de pacientes con infección respiratoria. Poco tiempo después se conoció su origen: una nueva pandemia que la OMS denominó COVID-19. Aunque no se ha demostrado científicamente su génesis, similares al coronavirus provienen y circulan entre los murciélagos; además, es altamente contagioso entre los seres humanos y genera consecuencias tales como la muerte (Trilla, 2020). Para la fecha, 22 de septiembre de 2020, se confirman en el mundo 31.377 .058 personas contagiadas y cerca de 965.681 muertes. Por tal razón, gobiernos de todo el mundo han implementado estrategias para prevenir el contagio masivo como el distanciamiento social, y el cierre de algunos sectores académicos y comerciales de alta afluencia.

Colombia, con una población cercana a 50 millones de habitantes, registra, para la misma fecha, 777.537 contagiados y 24.570 fallecidos. Dentro de las estrategias tomadas por el Gobierno nacional se puede mencionar el cierre de comercios y negocios de atención personalizada, escuelas, colegios y universidades, toque de queda, ley seca, prohibición de transporte fluvial, aéreo y terrestre, bajo algunas excepciones, con la finalidad de prevenir la propagación masiva del virus y un posterior colapso en los sistemas prestadores de servicio a la salud. Desde el primero de septiembre se pasó del aislamiento obligatorio a una fase denominada asilamiento selectivo, lo cual brinda la posibilidad de apertura paulatina de distintos sectores y vida social.

Pese a que la COVID-19, afecta directamente la salud de los individuos, debe ser comprendida también como una enfermedad social, que permite identificar y comprender las necesidades sociales, familiares e individuales que de allí emanan (Caraballeda, 2020). Sumado a los contextos particulares del país, como la desigualdad, pobreza, baja capacidad de acceso a servicios básicos, altos índices de trabajo informal, se hace necesario que las ayudas y estrategias que plantean entes locales, nacionales e internacionales puedan focalizar la población objetivo de los beneficios que pueden ofrecer (Rodríguez, 2020).

La pandemia y todas las consecuencias que trae consigo, generan entornos desfavorables para los seres humanos; por tal razón, la sociedad, mediante las distintas redes de apoyo, son de suma importancia para el acompañamiento mutuo, al igual que la comunicación constante, asertiva, vínculos afectivos y estables que hacen de las dinámicas familiares en tiempos de COVID-19 un refugio y espacio seguro para cada miembro (Vélez, Sánchez y Betancurth, 2020). La familia también es comprendida como un receptor de apoyos externos, para así poder mediar las múltiples tensiones que se genera directa o indirectamente de la enfermedad (Lozano, 2020).

Múltiples estudios e investigaciones sociales y de salud, resaltan la importancia de potenciar habilidades individuales, familiares y colectivas para afrontar las repercusiones negativas que puede traer la pandemia, el aislamiento social y otras medidas sanitarias (Ribot, Chang y González, 2020). Históricamente, el ser humano ha buscado y sentido la necesidad de otras personas; entre sus principales razones se encuentra la aceptación, afecto y apoyo (Bowlby, 
1969). Las familias son comprendidas como redes de apoyo para cada uno de sus integrantes, pero que a su vez necesita ayuda de otros entornos y sistemas, es así como las redes de apoyo se han fortalecido y han tenido mayor reconocimiento en las últimas décadas, gracias a sus aportes al desarrollo individual, familiar y social, lo que permite vislumbrar aún más la importancia del trabajo de las redes de apoyo en tiempos de pandemia.

En Colombia, según el Consejo de Estado (2011), se puede definir a la familia como una estructura social que se constituye a partir de un proceso que genera vínculos de consanguinidad o afinidad entre sus miembros. Por lo tanto, si bien la familia puede surgir como un fenómeno natural producto de la decisión libre de dos personas, lo cierto es que son las manifestaciones de solidaridad, fraternidad, apoyo, cariño, amor, lo que la estructuran y le brindan cohesión a la institución.

De las distintas posturas conceptuales, se puede indicar que la familia es una institución social, como indica Carol (2009), donde actualmente cobra una mayor relevancia para su análisis la construcción de redes de apoyo y el vínculo familiar; en este sentido, Rodríguez (2015), indica que la familia adquiere un papel importante en la cohesión social, que a su vez genera relaciones indispensables para el fortalecimiento de la convivencia humana. En cuanto a la concepción de la familia como vínculo familiar, Beytía (2018) señala que: "el vínculo familiar es un tipo específico de vínculo social, posiblemente el más primordial entre seres humanos" (p. 2).

Al igual que para la familia, y diversos conceptos sociales, difícilmente se podrá llegar a un consenso en la definición de las redes de apoyo por la complejidad de las mismas, y las infinitas maneras de interpretar, no solo en su significado, sino también en sus componentes y alcances, una de sus interpretaciones afirma que son un sistema para dar y recibir objetos, servicios, apoyo social y/o emocional (Lopata, 1975). Son también una serie de contactos que facilitan al ser humano una identidad social y que, a su vez, recibe apoyo emocional, material, servicios e información (Walker, MacBride y Vachon, 1977).

Es importante distinguir la fuente de las redes; la fuente primaria está constituida por la familia extensa, amigos, vecinos, etc., que están presentes en la vida cotidiana tanto pública como privada. Por su parte, la red secundaria o institucional, se refiere a las ofertas de apoyo que emanan de alguna institución, sin discriminar por su naturaleza (Desmaris, et. al, 1995). Por otra parte, se menciona que existen tres fuentes: la primera, está constituida por la red natural primaria y secundaria y allí se encuentra la familia extensa, amigos, vecinos, etc.; la segunda, son las organizaciones cívico-solidarias, que hacen referencia al apoyo informal que puedan recibir; por último, las instituciones sociales de apoyo formal (Lara, et. al, 2004).

Ahora bien, existe una categorización de los distintos tipos de apoyo, que a su vez son el sustento de análisis para la presente investigación: apoyo emocional, hace referencia a expresión, recepción de sentimientos y participación social en sus entornos, el apoyo tangible que menciona la ayuda material y el acompañamiento físico o ayuda en especie, por último, el apoyo informativo que destaca asuntos relacionados con datos e información de la actualidad, consejos y refuerzos positivos (López, et. Al, 2007).

Dentro de las diversas investigaciones realizadas sobre impactos psicosociales de la pandemia generada por la COVID-19 a distintos sectores de la sociedad, incluyendo a la familia, poco se ha mencionado acerca de la importancia de las redes de apoyo y la percepción que se tiene sobre ellas, es así como el presente artículo tiene como objetivo general conocer las redes de apoyo presentes en familias de Tunja durante la pandemia, como objetivos específicos se plantea indagar que es para las familias una red de apoyo e identificar cuáles son las redes de apoyo que más deberían estar presentes en tiempo de pandemia, según las familias participantes en la investigación. Sus resultados pueden contribuir a las distintas entidades y redes de apoyo existentes a realizar un análisis de su actuar en tiempos de crisis, igualmente, comprender la percepción y demandas de apoyo que tienen familias de la capital boyacense. 


\section{MÉTODO}

Diseño de Investigación. De acuerdo con el objetivo establecido, en la investigación se empleó un enfoque mixto, según Creswell y Plano (2007), es una estrategia de investigación o metodología donde el investigador, o el equipo de investigación, analiza, mezcla, integra o conecta datos cuantitativos y cualitativos en un único estudio, la recolección de la información cuantitativa y cualitativa se realizó de forma paralela. En concordancia, se utilizó el método descriptivo, resaltando que busca especificar las propiedades importantes de personas, grupos, comunidades o cualquier otro fenómeno que sea sometido a un análisis (Dankhe, 1986), en este caso particular, las redes de apoyo de familias de Tunja en tiempos de pandemia.

Participantes. Para la investigación, se contó con familias del municipio de Tunja, capital del departamento de Boyacá, la muestra fue de 119 participantes. Lo anterior se realizó, mediante muestreo no probabilístico por conveniencia, esta técnica de muestreo permite seleccionar aquellos casos accesibles que acepten ser incluidos, fundamentado en la conveniente accesibilidad y proximidad de los sujetos para el investigador (Tamara y Manterola 2017). Se buscaron participantes con distintas características sociodemográficas y se solicitó responder el instrumento en compañía de otro u otros miembros de cada familia, para así poder tener una visión integradora. Para la breve caracterización, se solicitaron datos de la persona identificada como jefe del hogar o en su defecto, la persona que tenía acceso al dispositivo donde se respondía el formulario.

Consideraciones Éticas. Al iniciar la aplicación del instrumento, se informó a los participantes que el uso de la información recolectada seria únicamente con fines investigativos, salvaguardando su identidad y posible información sensible allí expuesta.

Técnicas e Instrumentos. En esta investigación se empleó una encuesta, la cual es una técnica de investigación que recoge y analiza una serie de datos de una población que se pretende explorar, describir, predecir y/o explicar una serie de características. (García, 1993). Concordando con lo anterior, para la presente investigación se aplicó un cuestionario. Tamayo (2004), señala que es un instrumento que contiene los aspectos del fenómeno que se consideran esenciales, y permite aislar ciertos problemas que nos interesan, para este caso en particular, una breve caracterización de familias tunjanas, dificultades y fortalezas a nivel familiar durante la pandemia, apoyos y redes de apoyo presentes en tiempos de pandemia, así como el significado que le asignan y el conocimiento sobre la existencia de redes de apoyo a familias manifestado por los participantes. Su aplicación se desarrolló mediante la herramienta de Google Forms, y la divulgación del mismo, se realizó mediante el uso de redes sociales.

\section{Procedimiento}

Fase 1. Se realizó una revisión documental y estado del arte en temas relacionados a la pandemia generada por la COVID-19 y las medidas tomadas por el gobierno nacional, local para mitigar contagio masivo, simultáneamente, las incidencias que tiene en las dinámicas, relaciones al interior de las familias y la relación de ella con demás entornos.

Fase 2. Con base en la revisión documental, se crearon unas variables y categorías de análisis plasmadas en la Tabla 1, que permitieron dar paso a la elaboración de la metodología mixta de investigación y del instrumento.

Fase 3. En esta fase se llevó a cabo la divulgación y aplicación del instrumento a distintas familias de la ciudad, y se desarrolló del 27 de julio hasta el 30 de agosto de 2020.

Fase 4. Por último, se procedió al análisis de los datos; la información cuantitativa se plasmó en Tablas con su respectiva frecuencia y porcentaje, la información cualitativa se codificó para dar respuesta a las categorías presentadas, realizando también agrupación de las respuestas por similitud, con el fin de listar las de mayor presencia para su posterior análisis e interpretación. 
Bautista et al - Redes de apoyo a familias durante la pandemia: respuesta generada por la COVID-19

Tabla 1. Variables / Categorías

\begin{tabular}{cl}
\hline \multicolumn{1}{c}{ Variable } & \multicolumn{1}{c}{ Tabla de variables y categorías } \\
\hline \multirow{2}{*}{ Caracterización } & Sexo \\
& $\begin{array}{l}\text { Edad } \\
\text { Estrato socioeconómico }\end{array}$ \\
\hline \multirow{2}{*}{ Redes de apoyo } & Red con mayor presencia en tiempos de pandemia \\
& $\begin{array}{l}\text { Red que considera que debería estar más presente en tiempos de pandemia } \\
\text { Conocimiento de la existencia de redes de apoyo }\end{array}$ \\
\hline Tipo de apoyo & $\begin{array}{l}\text { Apoyo que más ha recibido en tiempos de pandemia } \\
\text { Apoyo que considera que debería estar más presente en tiempos de pandemia }\end{array}$ \\
\hline Categoría & Sortalezas familiares en tiempos de pandemia \\
\hline \multirow{2}{*}{ Entorno familiar } & Dificultades familiares en tiempos de pandemia \\
\hline \multirow{2}{*}{ Redes de apoyo } & $\begin{array}{l}\text { Significado de red de apoyo } \\
\text { Redes de apoyo en familias que conoce }\end{array}$ \\
\hline
\end{tabular}

Fuente: Elaboración propia, con base en la revisión documental.

\section{RESULTADOS}

A continuación, se presentan los resultados cuantitativos, cualitativos, plasmados en Tablas y narrativas respectivamente. La primera parte muestra una breve caracterización de las familias, seguido de un análisis DOFA que muestra las principales dificultades, oportunidades, fortalezas, amenazas familiares en tiempos de pandemia, redes de apoyo y los distintos apoyos presentes que solicitan los participantes en el mismo contexto generado por la COVID-19, el significado que le asignan a una red de apoyo, y por último las redes de apoyo a familias que conocen los participantes.

Tabla 2. Caracterización Sociofamiliar

\begin{tabular}{|c|c|c|}
\hline Variable & Frecuencia & Porcentaje \\
\hline \multicolumn{3}{|l|}{ Edad } \\
\hline De 11 a 19 años & 1 & $0.84 \%$ \\
\hline De 20 a 39 años & 78 & $65.5 \%$ \\
\hline De 40 a 64 años & 39 & $32.8 \%$ \\
\hline De 65 o más años & 1 & $0.84 \%$ \\
\hline \multicolumn{3}{|l|}{ Sexo } \\
\hline Mujer & 81 & $68.1 \%$ \\
\hline Hombre & 33 & $27.7 \%$ \\
\hline Prefiero no decirlo & 5 & $4.2 \%$ \\
\hline \multicolumn{3}{|c|}{ Estrato socioeconómico } \\
\hline 1 & 7 & $5.9 \%$ \\
\hline 2 & 43 & $36.1 \%$ \\
\hline 3 & 47 & $39.5 \%$ \\
\hline 4 & 18 & $15.1 \%$ \\
\hline 5 & 4 & $3.3 \%$ \\
\hline
\end{tabular}

Fuente: elaboración propia, con base en los resultados obtenidos. 
De acuerdo con la Tabla 2, las personas encuestadas se encuentran entre los 19 años, siendo este el límite inferior y 66 años, siendo el límite superior, cabe aclarar, que los participantes que oscilan entre el rango de 20 a 39 años son los de mayor presencia en la investigación, con una participación de 78 personas, representado en un $65.5 \%$, teniendo mayor frecuencia de participación con 22 años de edad. Pese a que se requería que dos o más miembros de cada familia participaran en la aplicación de cada encuesta, se solicitaba solo los datos para la caracterización de la persona jefe de hogar o, en su defecto, quien tuviese acceso al dispositivo donde se respondía el cuestionario. En ese orden de ideas, el $68.1 \%$ de los participantes son mujeres, que corresponde a 78 encuestadas, mientras que el $27.7 \%$ equivale a 33 hombres; por su parte, 5 personas, que representan un $4.2 \%$, prefieren no decirlo.

Para presentar los resultados de las principales fortalezas y dificultades de familias tunjanas en tiempos de pandemia, se realizó una codificación y posterior categorización en las 4 dimensiones del DOFA, que a su vez están divididas en dos: las fortalezas y debilidades, que se refieren a aspectos internos de la familia y las oportunidades y amenazas que mencionan factores externos a ellos.

Tabla 3. Análisis DOFA de familias en tiempos de pandemia.

\begin{tabular}{|c|c|c|}
\hline Variable & Fortaleza & Debilidad \\
\hline \multirow{12}{*}{ Interno } & Diálogo & Falta de actividades de ocio \\
\hline & Confianza & Falta de viajes \\
\hline & Unión & Falta de tiempo de calidad \\
\hline & Respeto & Regulación y manejo de tiempos \\
\hline & Comprensión & Falta de una apropiada comunicación \\
\hline & Resiliencia & Conflictos intrafamiliares \\
\hline & Autocuidado & Falta de privacidad \\
\hline & Buena comunicación & Diferencia de opiniones \\
\hline & & Dificultad alimentaria \\
\hline & & Distribución de las tareas del hogar \\
\hline & & Manejo del ruido en el hogar \\
\hline & & Encierro para niños y niñas \\
\hline \multirow{7}{*}{ Externo } & Oportunidades & Amenazas \\
\hline & Redes familiares de apoyo & Factores económicos \\
\hline & Cooperación & Desempleo \\
\hline & Estabilidad económica & Falta de apoyo gubernamental \\
\hline & Solidaridad & Carencia de atención en salud \\
\hline & Espiritualidad & Ausencia de trabajo estable \\
\hline & Acatar las directrices gubernamentales & \\
\hline
\end{tabular}

Fuente: elaboración propia, con base en los resultados obtenidos.

Para dar respuesta al factor interno, en las fortalezas manifestadas por las familias se identifican diálogo, confianza, unión, respeto, comprensión, resiliencia, auto cuidado, buena comunicación; por su parte, algunas de las principales debilidades encontradas son la falta de actividades de ocio, falta de viajes, falta de tiempo de calidad, regulación y manejo de tiempos, falta de una apropiada comunicación, el aumento de los conflictos intrafamiliares, la falta de privacidad, diferencia de opiniones, dificultad alimentaria, distribución de las tareas del hogar, el manejo del ruido, y el encierro para los niños y niñas.

Dentro del factor externo, compuesto en primer lugar por las oportunidades, las familias mencionaron los siguientes aspectos: las redes familiares de apoyo, cooperación, la estabilidad económica, solidaridad, espiritualidad y acatar directrices a nivel municipal, departamental y 
nacional. Por último, las principales amenazas manifestadas por las familias están relacionadas con factores económicos desfavorables, desempleo, falta de apoyo gubernamental, carencia de atención en salud y ausencia de trabajo estable.

En la Tabla 4 se evidencia que los participantes manifestaron que la red que más apoyo les ha brindado en tiempos de pandemia son Otros Familiares, con un $43.7 \%$, equivalente a 52 respuestas. De igual manera, es la misma red que ellos manifiestan que debería estar más presente en estas situaciones en particular con un $52.1 \%$. Identifican que la segunda red que más apoyo ha prestado a las familias participantes son los Amigos con un 32.8\%; en tercera posición, los resultados arrojan que 16 familias, que representan a un $13.4 \%$ manifiestan no haber recibido apoyo de ninguna red, en ese orden, continua la red de una Alguna Institución Pública o Privada con un 5.9\%, seguido por la Religión con un $2.5 \%$, por último la red que menos apoyo ha brindado a las familias participantes son los Vecinos, con un $1.7 \%$ equivalente a dos respuestas.

Tabla 4. Redes de Apoyo.

\begin{tabular}{lcc}
\hline \multicolumn{1}{c}{ Variable } & $\mathbf{N}$ & \% \\
\hline Red que más apoyo les ha brindado en tiempos de pandemia & $32.8 \%$ \\
Amigos & 39 & $43.7 \%$ \\
Otros familiares & 52 & $2.5 \%$ \\
Religión & 3 & $1.7 \%$ \\
Vecinos & 2 & $5.9 \%$ \\
Alguna institución (pública o privada) & 7 & $13.4 \%$ \\
Ninguna & 16 & $0 \%$ \\
Otros & 0 & $100 \%$ \\
TOTAL & 119 & $5.9 \%$ \\
\hline Red de apoyo que considera que debería estar más presente en tiempos de pandemia \\
\hline Amigos & 7 & $52.1 \%$ \\
Otros familiares & 62 & $1.7 \%$ \\
Religión & 2 & $1.7 \%$ \\
Vecinos & 2 & $34.4 \%$ \\
Alguna institución (pública o privada) & $3.4 \%$ \\
Ninguna & 41 & $0.8 \%$ \\
Otros & 4 & $100 \%$ \\
TOTAL & 119 & \\
\hline
\end{tabular}

Fuente: elaboración propia, con base en los resultados obtenidos.

Las redes institucionales (públicas o privadas), se encuentran en segundo lugar de la red que las familias consideran que debería estar más presente en tiempos de pandemia con un $34.4 \%$, las redes restantes ocupan un bajo porcentaje, los amigos con un 5.5\%, ninguna con 3.4\%, religión y vecinos con $1.7 \%$ cada una, por último, la opción otros, registra un $0.8 \%$, indicando que esta respuesta varía según la necesidad de cada familia.

Con la información plasmada en la Tabla 5, se presenta el apoyo que más han recibido las familias en tiempos de pandemia y cuál es el que ellas consideran que deberían estar más presente en momentos similares, el apoyo emocional se encuentra en primera posición en las dos variables, siendo el mayor apoyo presente con $55.5 \%$, frente a un $53.8 \%$ que respondieron que debería ser el apoyo de mayor aparición, en segundo lugar, el apoyo con mayor presencia es el informativo con un $18.5 \%$, seguido de ninguno con $13.4 \%$ que respecta a 16 familias, el apoyo tangible con $10.9 \%$, por último la opción otro con $1.6 \%$ donde mencionaron más de una opción. 
Tabla 5. Tipos de Apoyo.

\begin{tabular}{ccc}
\hline Variable & $\mathbf{N}$ & \% \\
\hline Apoyo que más ha recibido en tiempos de pandemia & & \\
\hline Emocional & 66 & $55.5 \%$ \\
Tangible & 13 & $10.9 \%$ \\
Informativo & 22 & $18.5 \%$ \\
Ninguno & 16 & $13.4 \%$ \\
Otro & 2 & $1.6 \%$ \\
TOTAL & 119 & $100 \%$ \\
\hline Apoyo que considera que debería estar más presente en tiempos de pandemia \\
\hline Emocional & 64 & $53.8 \%$ \\
Tangible & 41 & $34.5 \%$ \\
Informativo & 7 & $5.9 \%$ \\
Ninguno & 4 & $3.4 \%$ \\
Otro & 3 & $2.4 \%$ \\
\hline
\end{tabular}

Fuente: elaboración propia, con base en los resultados obtenidos.

Con respecto al apoyo que consideran que debería estar más presente en tiempos de pandemia, se encuentra en segundo lugar el apoyo tangible con 34.5\%, equivalente a 41 personas, seguido del informativo con un $5.9 \%$, ninguno con $3.4 \%$ y por último otro con $2.4 \%$, los cuales mencionaron que el tipo de ayuda que requieren varía según las necesidades y dinámicas de cada familia.

\section{Significado de una Red de Apoyo}

Con respecto a la pregunta ¿qué considera usted que es una red de apoyo?, se encontró similitud de respuestas; por ende, se procedió a organizar según afinidad. En primera instancia, lo relacionaron con una institución, entidad privada o pública, donde las personas acuden a buscar posibles soluciones frente a unas problemáticas específicas. Otra percepción estuvo ligada a un grupo de personas que ayudan a resolver problemas sociales específicos a una persona o familias que lo necesitan; dichas interpretaciones permiten identificar que las familias perciben a una red de apoyo como una institución o asociación de personas jurídicas o naturales que propende por el bienestar colectivo frente a la presencia de situaciones que los aquejan.

Por otra parte, mencionaron que también puede ser entendida como vínculos humanos que propician apoyo, pero que están cerca de nuestro entorno social y brindan orientación con el fin de enfrentar cualquier dificultad para tener una estabilidad emocional. En esta respuesta que se brindó, por parte de varias familias, se menciona la importancia de la estabilidad emocional y de los vínculos humanos dentro de la gestión, articulación con las diferentes redes de apoyo. Dicha interpretación no es ajena a la realidad, sino que, por el contrario, se complementa con las dos respuestas mencionadas anteriormente, permitiendo identificar las redes de apoyo, la articulación humana y social con ellas.

Conocimiento sobre Redes de Apoyo a Familias. En la Tabla 6, se identifica que un $71 \%$ equivalente a 84 personas, indican no conocer sobre redes de apoyo y un $29 \%$, equivalente a 35 personas, indican que sí. Los encuestados en general concordaron que una de las principales redes de apoyo que conocen están relacionadas con la iglesia, ICBF$^{1}$, ingreso solidario, Casa de la Mujer de la UPTC², comisarías de familia, entre otras.

${ }^{1}$ Instituto Colombiano de Bienestar Familia

¿Universidad Pedagógica y Tecnológica de Colombia 
Tabla 6. Conocimiento sobre Existencia de Redes de Apoyo a Familias.

\begin{tabular}{ccc}
\hline ¿Conoce usted la existencia de redes de apoyo a Familias? & N & \% \\
\hline No & 84 & $71 \%$ \\
Si & 35 & $29 \%$ \\
Total & 119 & $100 \%$ \\
\hline
\end{tabular}

Fuente: elaboración propia, con base en los resultados obtenidos.

\section{DISCUSIÓN}

El mayor porcentaje de participantes se encuentran en etapa de adultez temprana, caracterizada por la complejidad en las capacidades cognitivas, los juicios morales, en la toma de decisiones respecto a las relaciones íntimas y estilo vida. (Papalia, 2005). El ser humano durante esta etapa también posee cualidades sensoriales, psicomotrices que le permiten vivir con calidad y eficiencia, planteándose proyectos a mediano, a largo plazo, que incluyen a la pareja y a la familia. (Jiménez, 2012). Es así como esta etapa de vida es importante para poder hacer frente a los retos que trae por sí sola, y ahora sumado los retos de la pandemia, no solo como individuos, sino también como familia. Para la presente investigación la mayoría de personas consideradas jefes de hogar son mujeres. En Tunja, las familias con jefatura femenina equivales al $51 \%$ (SISBEN, 2012).

En lo que respecta al análisis del DOFA realizado, bajo la teoría de Max Neef (1986), se identifica que las necesidades de las familias en tiempos de cuarentena debido a la COVID-19 son de tipo axiológico y existencial, que se categorizan así: (ser/subsistencia); donde se identifica por ejemplo la salud en todos sus sentidos, (tener/protección); el empleo, los ingresos y la estabilidad laboral, (ser/ afecto); el manejo adecuado de emociones, resolución adecuada de conflictos, (hacer/entendimiento); capacidad de comprensión por parte de las familias frente a esta situación, (estar/participación); articulación con otros escenarios sociales, (hacer/creación); habilidades de la familias durante la COVID-19, (estar/identidad); pertenencia de la familia a un contexto social, (ser/libertad); autonomía, autoestima, voluntad, pasión, asertividad, apertura, determinación, audacia, tolerancia.

El análisis de las fortalezas y oportunidades de las familias en el contexto de la COVID-19, de acuerdo con su capacidad de búsqueda, identificación, activación de redes sociales de apoyo, no solo incide en la garantía de soluciones materiales e inmediatas, sino a su vez en el fortalecimiento de tejidos sociales familiares que durante la cuarentena y el aislamiento se debilitaron o disminuyeron. De acuerdo con Chamorro (2015), el desarrollo a escala humana propone como estrategia la autodependencia, pasando primero por el reconocimiento personal de las necesidades y satisfactores, fundamentado en las potencialidades en cada aspecto existencial: ser, tener, hacer, estar; estos reconocimientos personales permiten, potencian la independencia y autonomía, con el claro soporte de la unidad familiar.

En lo que respecta a la principal red de apoyo presente en tiempos de pandemia en familias de Tunja, concuerda con la red que creen que debería estar presente en situaciones similares, siendo otros familiares la que puntea en los resultados, otras investigaciones similares, por ejemplo, una realizada en México (Medellín et. Al, 2012), que presenta resultados similares a la presente, donde las dos redes con mayor presencia en situaciones de crisis familiares son otros familiares o familia extensa y los amigos, que se encuentra en segundo lugar de los resultados acá presentados, donde se manifiesta que se debe a óptimos niveles de cohesión familiar, proximidad y confianza.

El tipo de apoyo más recibido en tiempos de pandemia es el mismo que consideran que debería estar más presente en estos contextos, siendo el apoyo emocional el eje fundamental para los momentos adversos o de crisis para cada integrante de la familia y de ella en conjunto, en este caso, las repercusiones de la pandemia de la COVID-19, es importante mencionar que la red de apoyo familiar extensa o de otros familiares, es el pilar fundamental que brinda apoyo emocional a sus allegados (Cárdenas y Sebazco, 2000). 
Con respecto al significado que las familias participantes le asignan a una red de apoyo, se identifican varios aspectos. Se relaciona, en primera medida, a una red de apoyo informal porque se asocia con una organización burocrática, que contempla objetivos específicos en ciertas áreas determinadas y utiliza a profesionales o voluntarios para garantizar el logro de sus metas (Guzmán, Huenchuan y Montes 2003), relacionándola directamente con instrucciones o entidades públicas o privadas. Por otra parte, sigue siendo una red informal cuando lo relacionan con un grupo de personas diversas que presta ayuda en momentos difíciles; está conformada por un conjunto restringido no familiares, cuya función es brindar varias formas de apoyo, pueden disponer de ellas en situaciones críticas y fundamentalmente para desarrollar soluciones creativas frente a problemas como las generadas por las medidas sanitarias frente a la COVID-19 (Arias, 2009). Por último, algunas respuestas son pluralistas, porque incluyen miembros de la familia, amistades en las que se confía y que están dispuestas a ayudar.

Una de las principales respuestas en cuanto al conocimiento de redes de apoyo a familias, es la iglesia, teniendo en cuenta que Boyacá, su capital Tunja, es conocida por su fiel devoción y múltiples celebraciones religiosas, la religión puede ser comprendida como una institución jerárquica de alcance universal, que tiene una profunda influencia en procesos de carácter político, social y cultural que ocurren desde el espacio internacional hasta las pequeñas localidades. (Molina, 2012), en este caso las diversas estrategias que emplean para brindar apoyo emocional, tangible, entre otros. Mencionan otras redes institucionales que conforman una red informal, como por ejemplo las comisarías de familia que atienden casos de violencia intrafamiliar en cualquiera de sus tipologías y el ICBF, definida como la entidad del Estado colombiano que trabaja por la prevención y protección integral de la primera infancia, la niñez, la adolescencia y el bienestar de las familias en Colombia (Instituto Colombiano de Bienestar Familiar, 2019).

Por último, los encuestados resaltaron como red de apoyo el ingreso solidario, definido como un programa o un apoyo económico que estableció el Gobierno nacional a hogares en condición de pobreza, pobreza extrema y vulnerabilidad económica, para mitigar en dicha población los impactos derivados de la emergencia causada por la COVID-19 (Departamento Nacional de Planeación, 2020); de allí se puede inferir con base en la revisión de literatura, que el ingreso solidario puede ser entendido como un tipo de apoyo tangible, mientras que la red de apoyo seria el gobierno nacional, bajo la dependencia del Departamento Nacional de Planeación.

\section{CONCLUSIONES}

La familia extensa y los amigos se identifican como las principales redes de apoyo presentes en tiempos de pandemia generada por la COVID-19 a familias en Tunja, de allí surge la importancia y necesidad de apostar, trabajar por el fortalecimiento de las dinámica familiares y cohesión social, sin tener distinción por su tipología, extendiendo la invitación a tener una perspectiva desde el enfoque de género y diferencial, respetando así las múltiples manifestaciones de afecto, afinidad y organización en sociedad.

Lo anterior, implica una relevancia en la construcción de redes de apoyo para las familias de la ciudad de Tunja en el contexto de la COVID-19, dado que los planes, programas y proyectos que existan desde las políticas públicas sociales en familia deben pensar en atender las situaciones de las familias durante y después de la cuarentena, trabajando en identificación de aspectos de relevancia social, así como de manejo de crisis y emergencias sociales, de igual manera, es importante comenzar a crear estrategias que permitan potenciar los alcances de las redes de apoyo ya existentes, pues es considerable el número de participantes que manifestaron no haber recibido algún tipo de ayuda por alguna red de apoyo, y una de sus posibles razones, fundamentados en sus respuestas puede ser el desconocimiento de las mismas.

La sociedad civil, individual, colectiva, pública o privada se enfrenta a grandes retos durante y después de una crisis generada por la pandemia en la conexión como red de apoyo y el tipo de apoyo que oferta. De esta manera, no solo se deben priorizar variables económicas o necesidades materiales, sino a su vez necesidades humanas como el afecto, la escucha, la comprensión, la empatía, el respeto y la adaptación al cambio, ligado directamente con el tipo de 
ayuda emocional, recibido en mayor medida y el que más demanda en estos contextos, siendo de vital importancia para la salud mental individual, familiar y social. Es allí donde Trabajo Social, Ciencias Sociales y de la Salud, deben unir esfuerzos para procesos que van desde la elaboración de un diagnóstico social, creación de estrategias, vinculación, articulación, creación de redes apoyo, hasta la implementación, seguimiento y evaluación de ellas.

La investigación realizada en la ciudad de Tunja con las familias, permite señalar que en el contexto de la post cuarentena la cual lentamente empieza a materializarse y en lo que se ha denominado popularmente como la nueva normalidad, hace necesario repensar las relaciones entre inclusión social, población participante de programas y proyectos sociales y la redefinición de políticas públicas que identifiquen y reconozcan esa articulación entre la ciudadanía, la sociedad y el Estado, a través de las redes de apoyo, bien sean familiares, sociales e institucionales.

Se constituye, asimismo, para los profesionales en Trabajo Social, un objetivo común el apoyar, fomentar, fortalecer o participar de estas diversas redes de apoyo desde el reconocimiento de la dignidad humana, así como la promoción y protección de los derechos humanos de las familias. De esta manera, la investigación realizada permite concluir que el desafío de la post pandemia no solo será la recuperación económica, como lo han indicado analistas de la sociedad, sino también la reconstrucción y recuperación de tejidos sociales que tras meses de aislamiento generaron un distanciamiento físico y social, haciendo que quien intervenga en grupos poblacionales esté llamado a trabajar desde los lenguajes de redes de apoyo, solidaridad y justicia social.

\section{Conflicto de Intereses}

Los autores manifiestan no tener ningún conflicto de intereses.

\section{REFERENCIAS}

Arias, J. (2009). La red de apoyo social en la vejez: aportes para su evaluación. Revista de psicología da IMED, 1(1), 147-158.

Beytía, P. (2018). Vínculos familiares: una clave explicativa de la felicidad. En: Reyes, Carmen y Muñoz, Mónica. La familia en tiempos de cambio. Santiago: Ediciones UC. Recuperado: https://www.researchgate.net/publication/322798886

Bowlby, J. (1969). Attachment. Nueva York: Basic Books.

Carballeda, A. (2020). Trabajo Social en tiempos de pandemia [Audio podcast]. Recuperado de: https://www.margen.org/pandemia/carba_charla.html

Cárdenas, C.; Sebazco, A. (2000). Familia perdida. Características de esta crisis familiar. Revista Cubana de Medicina General Integral 16 (1), 93-97.

Carol, A. (2009). La familia como institución. I Congreso Internacional de Investigación y Práctica Profesional en Psicología XVI Jornadas de Investigación Quinto. Encuentro de Investigadores en Psicología del MERCOSUR. Facultad de Psicología - Universidad de Buenos Aires, Buenos Aires, Recuperado de: https://www.aacademica.org/000-020/606.pdf

Chamorro, A. (2015). Desarrollo a escala humana: óptica de vida de tres individuos con discapacidad. Equidad \& Desarrollo, 3 (24), 185-210. Recuperado de: https://dialnet.unirioja. es/descarga/articulo/5278699.pdf

Creswell., J.; Plano, V. (2007). El diseño y la realización de la investigación de métodos mixtos. Estados Unidos: Sage Publihsing. 
Dankhe, G. (1986). Investigación y comunicación: La comunicación humana: ciencia social, México: McGraw Hill.

Departamento Nacional de Planeación (2020) Manual operativo ingreso solidario. Recuperado: https://ingresosolidario.dnp.gov.co/documentos/Manual_Operativo-Ingreso-Solidario.pdf

Desmaris, D, et. Al. (1995). Paciente identificado, red primaria e ideología dominante: El campo de la intervención en salud mental. En: Elkaim, J, Las prácticas en la terapia de red. Barcelona: Gedisa.

García, F. (1993). La encuesta: en el análisis de la realidad social. Métodos y técnicas de Investigación. España: Alianza Universidad

Guzmán, M.; Huenchuan, S.; Montes, V. (2003). Redes de apoyo social de las personas mayores: Marco conceptual. Notas de población, 1(1) 35-65. Recuperado de: https://repositorio.cepal. org/bitstream/handle/11362/12750/np77035070 es.pdf?sequenc

Instituto Colombiano de Bienestar Familiar.(2019). ¿Que es el ICBF? Recuperado de: https:// www.icbf.gov.co/que-es-el-icbf

Jiménez, G. (2012). Teoría del desarrollo humano II. México DF, México: Red Tercer Milenio.

Lara, et. al. (2004). La influencia de los sucesos vitales y el apoyo social en una intervención psicoeducativa con mujeres con depresión. Salud Pública 46 (3), 378-387.

Lopata, H. (1979). Women As Widows: Support Systems. Nueva York: Elsevier.

López, et. al. (2007). Evaluación del apoyo social mediante la escala ASSIS: descripción y resultados a una muestra de madres en situación de riesgo psicosocial. Intervención Psicosocial 16 (3), 323-337.

Lozano, A. (2020). Impacto de la epidemia del Coronavirus (COVID-19) en la salud mental del personal de salud y en la población general de China. Revista de Neuro-Psiquiatría, 83(1), 51-56.

Max-Neef, M. (1986). Desarrollo a escala humana: Fundación Fundación Dag Hammarskjold. Recuperado de: http://www.daghammarskjold.se/wp-content/uploads/1986/08/86 especial.pdf

Medellín, et al. (2012). Funcionamiento familiar y su relación con las redes de apoyo social en una muestra de Morelona, México. Salud Mental 35 (3), 147-154. Recuperado de: http:// www.scielo.org.mx/scielo.php?script=sci_arttext\&pid=S0185-33252012000200008

Molina, M. (2012). La Iglesia católica en el espacio público: un proceso de continua adecuación. Política y cultura, 2(38), 49-65. Recuperado de: http://www.scielo.org.mx/ scielo.php?script $=$ sci arttext\&pid=S0188-77422012000200004

Papalia, E. (2005) Desarrollo humano, Sao Pablo, Brasil: McGraw-Hill. Recuperado de: https:// repositorioinstitucional.ceu.es/bitstream/10637/2315/2/Cap.\%201\%20-\%20Acerca\%20 del\%20desarrollo\%20humano\%28P\%c3\%a1ginas\%206-23\%29.pdf

Ribot, V.; Chang, N.; Lázaro, A. (2020). Efectos de la COVID-19 en la salud mental de la población. Revista Habanera de Ciencias Médicas 2(19), 1-11. Recuperado de: http://www. revhabanera.sld.cu/index.php/rhab/article/view/3307

Rodríguez, Á. (2015). Redes familiares, estrategias de resiliencia e intervenciones colaborativas en trabajo social comunitario. Revista Familia 51 (2), 31-42. Recuperado: https://core. ac.uk/download/pdf/50606721.pdf 
Rodríguez, E. (2020). Colombia. Impacto económico, social y político de la COVID-19. Análisis Carolina 1-14. https://doi.org/10.33960/AC 24.2020

SISBEN. (2012). población y jefatura femenina de hogar.

Tamara., O.; Manterola, C. (2017). Técnicas de Muestreo sobre una Población a Estudio. International Journal of Morphology, 35(1), 227-232. Recuperado de: https://scielo.conicyt.cl/scielo. php?script $=$ sci arttext $\&$ pid $=$ S0717-95022017000100037

Tamayo, M. (2004). El proceso de la investigación científica, Ciencias Sociales, México: Limusa Noriega.

Consejo de estado Colombia. (2013). Sentencia 2001-00757 del 9 de julio de 2013. Recuperado de: http://www.lexbasesa.com/FrontPageLex/libreria/cl0003/2-18701-consejo-de-esatdo--concepto-de-familia-mp-enrique-gil-botero-familia-consejo-de-estado.htm

Trilla, A. (2020). Un mundo, una salud: la epidemia por el nuevo coronavirus COVID-19. Medicina Clínica 154(5) 175-177. Recuperado de: https://www.sciencedirect.com/science/article/pii/ S002577532030141X?via\%3Dihub

Vélez, C.; Sánchez, N.; Betancurth, L. (2020). Cuarentena por COVID-19 en un profesional de la salud: dimensión psicológica, social y familar. Salud Pública 22 (2) 1-5.

Walker, K.; MacBride, A. ; Vachon, M. (1977). Social support networks and the crisis of bereavement, Social Science and Medecine 11 (1), 35-41. https://doi.org/10.1016/0037-7856(77)90143-3 